\title{
BALANCED METRICS AND CHOW STABILITY OF PROJECTIVE BUNDLES OVER RIEMANN SURFACES
}

\author{
REZA SEYYEDALI
}

(Communicated by Michael Wolf)

\begin{abstract}
In 1980, I. Morrison proved that slope stability of a vector bundle of rank 2 over a compact Riemann surface implies Chow stability of the projectivization of the bundle with respect to certain polarizations. In a previous work, we generalized Morrison's result to higher rank vector bundles over compact algebraic manifolds of arbitrary dimension that admit a constant scalar curvature metric and have a discrete automorphism group. In this article, we give a simple proof for polarizations $\mathcal{O}_{\mathbb{P} E^{*}}(d) \otimes \pi^{*} L^{k}$, where $d$ is a positive integer, $k \gg 0$ and the base manifold is a compact Riemann surface of genus $g \geq 2$.
\end{abstract}

\section{INTRODUCTION}

In [M], Morrison proved that for the projectivization of a rank two holomorphic vector bundle over a compact Riemann surface, Chow stability is equivalent to the stability of the bundle. In $\underline{\underline{S}}$, we generalized one direction of Morrison's result for higher rank vector bundles over compact algebraic manifolds of arbitrary dimension that admit a constant scalar curvature metric and have a discrete automorphism group.

Let $X$ be a compact complex manifold of dimension $m$ and $\pi: E \rightarrow X$ be a holomorphic vector bundle of rank $r$ with dual bundle $E^{*}$. This gives a holomorphic fibre bundle $\mathbb{P} E^{*}$ over $X$ with fibre $\mathbb{P}^{r-1}$. We denote the tautological line bundle on $\mathbb{P} E^{*}$ by $\mathcal{O}_{\mathbb{P} E^{*}}(-1)$ and its dual by $\mathcal{O}_{\mathbb{P} E^{*}}(1)$. Let $L \rightarrow X$ be an ample line bundle on $X$ and $\omega \in 2 \pi c_{1}(L)$ be a Kähler form. Since $L$ is ample, there is an integer $k_{0}$ so that for any $k \geq k_{0}, \mathcal{O}_{\mathbb{P} E_{k}^{*}}(1)$ is very ample over $\mathbb{P} E_{k}^{*}$, where $E_{k}=E \otimes L^{\otimes k}$. Note that there is a canonical isomorphism $\mathbb{P} E_{k}^{*} \cong \mathbb{P} E^{*}$ and $\mathcal{O}_{\mathbb{P} E_{k}^{*}}(1) \cong \mathcal{O}_{\mathbb{P} E^{*}}(1) \otimes \pi^{*} L^{k}$. The main theorem of $[\underline{S}]$ is the following:

Theorem 1.1. Suppose that $A u t(X)$ is discrete and $X$ admits a constant scalar curvature Kähler metric in the class of $2 \pi c_{1}(L)$. If $E$ is Mumford stable, then

$$
\left(\mathbb{P} E^{*}, \mathcal{O}_{\mathbb{P} E^{*}}(1) \otimes \pi^{*} L^{k}\right)
$$

is Chow stable for $k \gg k_{0}$.

One of the earliest results in this spirit is the work of Burns and De Bartolomeis in $[\mathrm{BD}$. They constructed a ruled surface which does not admit any extremal metric in a certain cohomology class. In [H1], Hong proved that there are constant scalar curvature Kähler metrics on the projectivization of stable bundles over

Received by the editors November 28, 2010 and, in revised form, November 11, 2011.

2010 Mathematics Subject Classification. Primary 32Q26; Secondary 53C07. 
curves. In [H2] and [H3], he generalized this result to higher dimensions with some extra assumptions. Combining Hong's results with Donaldson's Theorem (D3. Theorem 3]), $\left(\mathbb{P} E^{*}, \mathcal{O}_{\mathbb{P} E^{*}}(n) \otimes \pi^{*} L^{m}\right)$ is Chow stable for $n \gg m \gg 0$ when the bundle $E$ is stable. Note that it differs from our result, since it implies the Chow stability of $\left(\mathbb{P} E^{*}, \mathcal{O}_{\mathbb{P} E_{m}^{*}}(n)\right)$ for $n \gg m \gg 0$.

In RT, Ross and Thomas developed the notion of slope stability for polarized algebraic manifolds. As one of the applications of their theory, they proved that if $\left(\mathbb{P} E^{*}, \mathcal{O}_{\mathbb{P} E^{*}}(1) \otimes \pi^{*} L^{k}\right)$ is slope semistable for $k \gg 0$, then $E$ is a slope semistable bundle and $(X, L)$ is a slope semistable manifold. Again note that they look at stability of $\mathbb{P} E^{*}$ with respect to polarizations $\mathcal{O}_{\mathbb{P} E_{m}^{*}}(n)$ for $n$ large enough. For the case of one dimensional base, however they showed stronger results. In this case they proved that if $\left(\mathbb{P} E^{*}, \mathcal{O}_{\mathbb{P} E^{*}}(1) \otimes \pi^{*} L\right)$ is slope (semi, poly) stable for any ample line bundle $L$, then $E$ is a slope (semi, poly) stable bundle.

In order to prove Theorem 1.1 we use the concept of balanced metrics. Combining the results of Luo, Phong, Sturm, Wang and Zhang on the relation between balanced metrics and stability, we prove the following.

Theorem $1.2([\underline{S}])$. Suppose that $A u t(X)$ is discrete and $X$ admits a constant scalar curvature Kähler metric in the class of $2 \pi c_{1}(L)$. If $E$ is Mumford stable, then

$$
\left(\mathbb{P} E^{*}, \mathcal{O}_{\mathbb{P} E^{*}}(1) \otimes \pi^{*} L^{k}\right)
$$

admits balanced metrics for $k \gg 0$.

In this paper, we give another proof of Theorem 1.1 in the case of a one dimensional base $X$. The proof is simple in this case and can be generalized to polarizations $\mathcal{O}_{\mathbb{P} E^{*}}(d) \otimes \pi^{*} L^{k}$ for any positive integer $d$ and $k \gg 0$. The main theorem of this paper is the following.

Theorem 1.3. Let $X$ be a compact Riemann surface of genus $g \geq 2$ and $E \rightarrow X$ be a holomorphic vector bundle on $X$. Let $d$ be a positive integer. If $E$ is Mumford stable, then

$$
\left(\mathbb{P} E^{*}, \mathcal{O}_{\mathbb{P} E^{*}}(d) \otimes \pi^{*} L^{k}\right)
$$

admits balanced metrics $g_{k}$ for $k \gg 0$.

The Hitchin-Kobayashi correspondence implies that the stable bundle $E$ admits a Hermitian-Einstein metric $h_{\infty}$. A simple calculation shows that the Hermitian metric $\operatorname{Sym}^{d} h_{\infty}$ on $\operatorname{Sym}^{d} E$ is Hermitian-Einstein. Therefore the vector bundle $\mathrm{Sym}^{d} E$ is stable. By a theorem of Wang, we know that there exist balanced metrics $H^{(k)}$ on $\operatorname{Sym}^{d} E \otimes L^{k}([\mathrm{~W} 2])$. This means that there exists a basis $s_{1}, \ldots, s_{N}$ for $H^{0}\left(X, \operatorname{Sym}^{d} E \otimes L^{k}\right)$ such that

$$
\begin{aligned}
& \sum_{i} s_{i} \otimes s_{i}^{*^{(k)}}=I_{\mathrm{Sym}^{d} E}, \\
& \int_{X}\left\langle s_{i}, s_{j}\right\rangle_{H^{(k)}} \omega=\frac{r V}{N} \delta_{i j} .
\end{aligned}
$$

Using the canonical isomorphism between $H^{0}\left(X, \operatorname{Sym}^{d} E \otimes L^{k}\right)$ and $H^{0}\left(\mathbb{P} E^{*}\right.$, $\left.\mathcal{O}_{\mathbb{P} E^{*}}(d) \otimes L^{k}\right)$, we get a sequence of Hermitian metrics $\widehat{H^{(k)}}$ on $\mathcal{O}_{\mathbb{P} E^{*}}(d) \otimes L^{k}$. On the other hand, Wang also proved that if $\omega$ is a $\operatorname{cscK}$ metric, then the sequence of rescaled balanced metrics converges to the Hermitian-Einstein metric $\mathrm{Sym}^{d} h_{\infty}$ on $\operatorname{Sym}^{d} E$ ( $[\mathrm{W} 2]$. In general the rate of convergence is $O\left(k^{-1}\right)$. However, in the 
case that the base manifold is a Riemann surface, it is easy to show that the rate of convergence is $O\left(k^{-\infty}\right)$ (cf. Theorem 3.5). This enables us to prove that the sequence $\widehat{H^{(k)}}$ is "almost balanced", i.e.

$$
\int\left\langle\widehat{s}_{i}, \widehat{s_{j}}\right\rangle_{\widehat{H^{(k)}}} d v o l_{\widehat{h^{(k)}}}=D^{(k)} \delta_{i j}+M_{i j}^{(k)},
$$

where $D^{(k)} \rightarrow C_{r, d}$ as $k \rightarrow \infty$ (see (2.2) for the definition of $C_{r, d}$ ) and $M^{(k)}$ is a trace-free Hermitian matrix such that $\left\|M^{(k)}\right\|_{o p}=O\left(k^{-\infty}\right)$ as $k \rightarrow \infty$.

The next step is to perturb these almost balanced metrics to get balanced metrics. As pointed out by Donaldson, the problem of finding a balanced metric can also be viewed as a finite dimensional moment map problem solving the equation $M^{(k)}=0$. Indeed, Donaldson shows that $M^{(k)}$ is the value of a moment map $\mu_{D}$ on the space of ordered bases with the obvious action of $S U(N)$. Now, the problem is to show that if for some ordered basis $\underline{s}$ the value of a moment map is very small, then we can find a basis at which a moment map is zero. The standard technique is flowing down $\underline{s}$ under the gradient flow of $\left|\mu_{D}\right|^{2}$ to reach a zero of $\mu_{D}$. We need a Lojasiewicz type inequality to guarantee that the flow converges to a zero of the moment map. This was done in $[\mathbf{S}$ ] by adapting the Phong-Sturm proof to our situation. In [S2], we generalize Theorem 1.3 to higher dimensional base manifolds that admit cscK metrics and do not have any nonzero holomorphic vector fields. After this work was completed, we became aware of the preprint [DZ].

\section{Preliminaries}

Let $V$ be a Hermitian vector space of dimension $r$. The projective space $\mathbb{P} V^{*}$ can be identified with the space of hyperplanes in $V$ via

$$
f \in V-\{0\} \rightarrow \operatorname{ker}(f)=V_{f} \subset V .
$$

There is a natural isomorphism between $V$ and $H^{0}\left(\mathbb{P} V^{*}, \mathcal{O}_{\mathbb{P} V^{*}}(1)\right)$ which sends $v \in V$ to $\hat{v} \in H^{0}\left(\mathbb{P} V^{*}, \mathcal{O}_{\mathbb{P} V^{*}}(1)\right)$ such that for any $f \in V^{*}, \hat{v}(f)=f(v)$. Any Hermitian inner product $h$ on $V$ induces a Hermitian inner product $\widehat{h}$ on $\mathcal{O}_{\mathbb{P} V^{*}}(1)$ as follows:

for $v, w \in V$ and $f \in V^{*}$.

$$
\langle\hat{v}, \hat{w}\rangle_{\widehat{h}}(f)=\frac{f(v) \overline{f(w)}}{|f|^{2}}
$$

For any positive integer $d$, define an equivalence relation $\sim$ on $V^{\otimes d}$ by

$$
v_{1} \otimes \cdots \otimes v_{d} \sim v_{\sigma(1)} \otimes \cdots \otimes v_{\sigma(d)}, \quad \sigma \in S_{d} .
$$

We define $\operatorname{Sym}^{d} V=V^{\otimes d} / \sim$ and simply denote the class of $v_{1} \otimes \cdots \otimes v_{d}$ in $\operatorname{Sym}^{d} V$ by $v_{1} \ldots v_{d}$. Similar to the case of $d=1$ any Hermitian inner product $h$ on $V$ induces a Hermitian inner product $\mathrm{Sym}^{d} h$ on $\operatorname{Sym}^{d} V$ by

$$
\left\langle v_{1} \ldots v_{d}, w_{1} \ldots w_{d}\right\rangle_{\mathrm{Sym}^{d} h}=\frac{1}{d !} \sum_{\sigma \in S_{d}}\left\langle v_{1}, w_{\sigma(1)}\right\rangle_{h} \ldots\left\langle v_{d}, w_{\sigma(d)}\right\rangle_{h} .
$$

Remark 2.1. Let $e_{1}, \ldots, e_{r}$ be an orthonormal basis for $V$ with respect to $h$. Then the set

$$
\left\{\left(\frac{i_{1} ! \ldots i_{r} !}{d !}\right)^{\frac{-1}{2}} e_{1}^{i_{1}} \ldots e_{r}^{i_{r}} \mid 0 \leq i_{\alpha} \leq d, \sum_{\alpha=1}^{r} i_{\alpha}=d\right\}
$$

forms an orthonormal basis for $\operatorname{Sym}^{d} V$ with respect to $\mathrm{Sym}^{d} h$. 
There is a natural isomorphism between $\operatorname{Sym}^{d} V$ and $H^{0}\left(\mathbb{P} V^{*}, \mathcal{O}_{\mathbb{P} V^{*}}(d)\right)$ which sends $v_{1} \ldots v_{d} \in \operatorname{Sym}^{d} V$ to ${\widehat{v_{1} \ldots v_{d}}}_{d} \in H^{0}\left(\mathbb{P} V^{*}, \mathcal{O}_{\mathbb{P} V^{*}}(d)\right)$ defined by

$$
{\widehat{v_{1} \ldots v_{d}}}_{d}\left(\left[v^{*}\right]\right)\left(w_{1}^{*} \otimes \cdots \otimes w_{d}^{*}\right)=w_{1}^{*}\left(v_{1}\right) \ldots w_{d}^{*}\left(v_{d}\right)
$$

where $v^{*} \in V^{*}-\{0\}$ and $w_{i}^{*} \in V^{*}$ are scalar multiples of $v^{*}$. There exist complex numbers $\lambda_{1}, \ldots, \lambda_{d}$ such that $w_{i}^{*}=\lambda_{i} v^{*}$. Thus,

$$
\widehat{v_{1} \ldots v_{d}}\left(\left[v^{*}\right]\right)\left(w_{1}^{*} \otimes \cdots \otimes w_{d}^{*}\right)=\lambda_{1} \ldots \lambda_{d} v^{*}\left(v_{1}\right) \ldots v^{*}\left(v_{d}\right),
$$

and therefore (2.1) defines a well-defined section of $\mathcal{O}_{\mathbb{P} V^{*}}(d)$.

For any Hermitian inner product $H$ on $\operatorname{Sym}^{d} V$, we define a metric $\hat{H}$ on $\mathcal{O}_{\mathbb{P} V^{*}}(d)$ by

In particular, we have

$$
\langle\hat{s}, \hat{t}\rangle_{\hat{H}}[v]=\frac{v^{\otimes d}(s) \overline{v^{\otimes d}(t)}}{|v \ldots v|_{H}^{2}} .
$$

$$
\langle\hat{s}, \hat{t}\rangle_{\widehat{\operatorname{Sym}^{d} h}}[v]=\frac{v^{\otimes d}(s) \overline{v^{\otimes d}(t)}}{|v|_{h}^{2 d}} .
$$

The following lemmas are straightforward.

Lemma 2.2. For any Hermitian inner product $h$ on $V$, we have

$$
\hat{h}^{\otimes d}=\widehat{\operatorname{Sym}^{d} h} .
$$

Lemma 2.3. There exists a constant $C_{r, d}$ such that for any $v, w \in \operatorname{Sym}^{d} V$ and any Hermitian inner product $h$ on $V$,

$$
d^{r-1} \int_{\mathbb{P}^{*}}\langle\hat{v}, \hat{w}\rangle_{\widehat{S y m^{d} h}} \frac{\omega_{F S, h}^{r-1}}{(r-1) !}=C_{r, d}\langle v, w\rangle_{S y m^{d} h},
$$

where $\omega_{F S, h}=i \bar{\partial} \partial \log \hat{h}$.

Remark 2.4. Let $H$ be a Hermitian inner product on $\mathrm{Sym}^{d} V$. Suppose there exists a constant $C$ such that

$$
\int_{\mathbb{P} V^{*}}\langle\hat{v}, \hat{w}\rangle_{\widehat{H}} \frac{\omega_{\mathrm{FS}, H}^{r-1}}{(r-1) !}=C\langle v, w\rangle_{H},
$$

for any $v, w \in \operatorname{Sym}^{d} V$. Then $H=\operatorname{Sym}^{d} h$ for some Hermitian inner product $h$ on $V$.

Lemma 2.5. Let $h_{0}$ and $h$ be Hermitian inner products on $V$. If $\left\|h-h_{0}\right\| h_{h_{0}} \leq \epsilon$, then $\left\|\hat{h}-\hat{h}_{0}\right\|_{C^{2}\left(\hat{h}_{0}\right)} \leq C \epsilon$ for a constant $C$ that depends only on $r$ and $d$.

Lemma 2.6. Let $X$ be a Kähler manifold of dimension $n$ and $\Omega_{0}$ and $\Omega$ be two $K$ ähler forms on $X$. There exists a constant $C$ that depends only on the dimension of $X$ such that if $\left\|\Omega-\Omega_{0}\right\|_{C^{0}\left(\Omega_{0}\right)} \leq \epsilon$, then $\left|\frac{\Omega^{n}-\Omega_{0}^{n}}{\Omega_{0}^{n}}\right| \leq C \epsilon$.

Proposition 2.7. Let $h$ be a Hermitian inner product on $V$ and $H$ be a Hermitian inner product on $\operatorname{Sym}^{d} V$ such that $\left\|H-\operatorname{Sym}^{d} h\right\|_{S y m^{d} h}<\min \left(\epsilon, \frac{1}{2}\right)$. Then for any $v, w \in \operatorname{Sym}^{d} V$, we have

$$
\left|d^{r-1} \int_{\mathbb{P} V^{*}}\langle\widehat{v}, \widehat{w}\rangle_{\widehat{H}} \frac{\omega_{F S, h}^{r-1}}{(r-1) !}-C_{r, d}\langle v, w\rangle_{H}\right| \leq C \epsilon|v|_{H}|w|_{H},
$$

where $C$ is a constant that depends only on $r$ and $d$. 
Proof. Let $e_{1}, \ldots, e_{K}$ be an orthonormal basis for $\operatorname{Sym}^{d} V$ with respect to $\operatorname{Sym}^{d} h$. Define $H_{i j}=H\left(e_{i}, e_{j}\right)$ and $\epsilon_{i j}=H_{i j}-\delta_{i j}$. We have $\left|\epsilon_{i j}\right|=\left|H_{i j}-\delta_{i j}\right| \leq \epsilon$. Given $v, w \in \operatorname{Sym}^{d} V$, we can write $v=\sum a_{i} e_{i}$ and $w=\sum b_{i} e_{i}$. We have

$$
\begin{aligned}
\left|\langle v, w\rangle_{\mathrm{Sym}^{d} h}-\langle v, w\rangle_{H}\right| & =\left|\sum a_{i} \overline{b_{i}}-\sum a_{i} \overline{b_{j}} H_{i j}\right|=\left|\sum a_{i} \overline{b_{j}} \epsilon_{i j}\right| \\
& \leq \epsilon \sum\left|a_{i}\right|\left|\overline{b_{j}}\right| \leq K \epsilon \sum\left|a_{i}\right|^{2} \sum\left|b_{j}\right|^{2} \\
& =\epsilon K|v|_{\operatorname{Sym}^{d} h}|w|_{\operatorname{Sym}^{d} h} .
\end{aligned}
$$

The last inequality follows from Cauchy-Schwartz inequality. By a unitary change of basis we may assume that $H_{i j}=0$ if $i \neq j$. Therefore the basis $\left\{f_{1}=\right.$ $\left.H_{11}^{\frac{-1}{2}} e_{1}, \ldots, f_{K}=H_{11}^{\frac{-1}{2}} e_{K}\right\}$ is an orthonormal basis for $\operatorname{Sym}^{d} V$ with respect to $H$. We have $\frac{1}{2} \leq H_{i i} \leq \frac{3}{2}$ since $\left|H_{i i}-1\right| \leq \frac{1}{2}$. Thus,

$$
\left.|| f_{i}\right|_{\text {Sym }^{d} h} ^{2}-\left|f_{i}\right|_{H}^{2}|=| 1-H_{i i}^{-1} \mid=\frac{\left|1-H_{i i}\right|}{H_{i i}} \leq 2 \epsilon .
$$

Therefore by the same argument, we conclude that

$$
\left|\langle v, w\rangle_{\mathrm{Sym}^{d} h}-\langle v, w\rangle_{H}\right| \leq 2 \epsilon K|v|_{H}|w|_{H} .
$$

Applying (2.3), Lemma 2.3. Lemma 2.5 and Lemma 2.6, we have

$$
\begin{aligned}
& \left|d^{r-1} \int_{\mathbb{P} V^{*}}\langle\widehat{v}, \widehat{w}\rangle_{\widehat{H}} \frac{\omega_{F S, h}^{r-1}}{(r-1) !}-C_{r, d}\langle v, w\rangle_{H}\right| \\
& \leq\left|d^{r-1} \int_{\mathbb{P}^{*}}\langle\widehat{v}, \widehat{w}\rangle_{\widehat{H}} \frac{\omega_{F S, h}^{r-1}}{(r-1) !}-d^{r-1} \int_{\mathbb{P}^{*}}\langle\widehat{v}, \widehat{w}\rangle_{\operatorname{Sym}^{d} h} \frac{\omega_{F S, h}^{r-1}}{(r-1) !}\right| \\
& +C_{r, d}\left|\langle v, w\rangle_{\mathrm{Sym}^{d} h}-\langle v, w\rangle_{H}\right| \\
& \leq d^{r-1} \int_{\mathbb{P} V^{*}}\left|\langle\widehat{v}, \widehat{w}\rangle_{\widehat{H}}-\langle\widehat{v}, \widehat{w}\rangle_{\operatorname{Sym}^{d} h}\right| \frac{\omega_{F S, h}^{r-1}}{(r-1) !}+C_{r, d}\left|\langle v, w\rangle_{\operatorname{Sym}^{d} h}-\langle v, w\rangle_{H}\right| \\
& \leq C \epsilon d^{r-1} \int_{\mathbb{P V}^{*}}|\widehat{v}|_{\operatorname{Sym}^{d} h}|\widehat{w}|_{\operatorname{Sym}^{d} h} \frac{\omega_{F S, h}^{r-1}}{(r-1) !}+2 K C_{r, d} \epsilon|v|_{H}|w|_{H} \\
& \leq \epsilon\left(C d^{r-1} V+2 K C_{r, d}\right)|v|_{H}|w|_{H} .
\end{aligned}
$$

The last inequality follows from the fact that $\sup _{\mathbb{P} V^{*}}|\widehat{v}|_{\operatorname{Sym}^{d} h}=|v|_{\operatorname{Sym}^{d} h}$.

\section{BALANCED METRICS ON HOLOMORPHIC VECTOR BUNDLES}

Let $\left(X, \omega_{0}\right)$ be a compact Kähler manifold of dimension $n$ and $(L, g)$ be an ample holomorphic Hermitian line bundle over $X$ such that $i \bar{\partial} \partial \log g=\omega_{0}$. Let $E$ be a holomorphic vector bundle of rank $r$ over $X$. By possibly tensoring with high power of the ample line bundle $L$, we may assume that $E$ is very ample. Therefore we can embed $X$ into $G\left(r, H^{0}(X, E)^{*}\right)$, the Grassmanian of $r$-planes in $H^{0}(X, E)^{*}$. Indeed, for any $x \in X$, we have the evaluation map $H^{0}(X, E) \rightarrow E_{x}$, which sends $s$ to $s(x)$. Since $E$ is globally generated, this map is a surjection. So its dual is an inclusion of $E_{x}^{*} \hookrightarrow H^{0}(X, E)^{*}$, which determines an $r$-dimensional subspace of $H^{0}(X, E)^{*}$. Therefore we get a map $\iota: X \rightarrow G\left(r, H^{0}(X, E)^{*}\right)$. Since $E$ is very ample, $\iota$ is an embedding. Clearly we have $\iota^{*} U_{r}=E^{*}$, where $U_{r}$ is the tautological vector bundle on $G\left(r, H^{0}(X, E)^{*}\right)$; i.e. at any $r$-plane in $G\left(r, H^{0}(X, E)^{*}\right)$, the fibre of $U_{r}$ is 
exactly that $r$-plane. A choice of basis for $H^{0}(X, E)$ gives an isomorphism between $G\left(r, H^{0}(X, E)^{*}\right)$ and the standard Grassmanian $G(r, N)$, where $N=\operatorname{dim} H^{0}(X, E)$. We have the standard Fubini-Study Hermitian metric on $U_{r}$, so we can pull it back to $E$ and get a Hermitian metric on $E$.

Definition 3.1. The embedding is called balanced if

$$
\int_{X}\left\langle s_{i}, s_{j}\right\rangle_{\iota^{*} h_{\mathrm{FS}}} \frac{\omega^{n}}{n !}=C \delta_{i j} .
$$

Notice that being balanced depends on the choice of the Kähler form. A Hermitian metric on $E$ is called balanced (more precisely $\omega$-balanced) if it is the pullback $\iota^{*} h_{\mathrm{FS}}$, where $\iota$ is a balanced embedding.

Equivalently, we can formulate the definition of balance metrics in terms of Bergman kernels.

Definition 3.2. Let $h$ be a Hermitian metric on $E$ and $s_{1}, \ldots, s_{N}$ be an orthonormal basis for $H^{0}(X, E)$ with respect to the inner product

$$
\langle s, t\rangle=\int_{X}\langle s(x), t(x)\rangle_{h} \frac{\omega_{0}^{n}}{n !} .
$$

The Bergman kernel of $(E, h)$ is an endomorhism of $E$ defined by

$$
B\left(h, \omega_{0}\right)=\sum_{i=1}^{N} s_{i} \otimes s_{i}^{*_{h}} .
$$

Note that $B\left(h, \omega_{0}\right)$ does not depend on the choice of the orthonormal basis.

A Hermitian metric $h$ on $E$ is balanced if and only if $B\left(h, \omega_{0}\right)=C I_{E}$ for a positive constant $C$.

We recall the Catlin-Tian-Yau-Zeldich asymptotic expansion of the Bergman kernel.

Theorem $3.3\left([\mathrm{C},[\mathrm{Z}])\right.$. Let $\left(X, \omega_{0}\right)$ be a compact Kähler manifold of dimension $n$ and $(L, g)$ be an ample holomorphic Hermitian line bundle over $X$ such that $i \bar{\partial} \partial \log g=\omega_{0}$. For any Hermitian metric $h$ on the vector bundle $E$, there exist smooth endomorphisms $A_{i}(h) \in \Gamma(X, E n d(E))$ such that the following asymptotic expansion holds as $k \rightarrow \infty$ :

$$
B\left(h \otimes g^{\otimes k}, \omega_{0}\right) \sim k^{n}+A_{1}(h) k^{n-1}+\ldots .
$$

There is a close relationship between stability of vector bundles and the existence of balanced metrics given by the following theorem of Wang.

Theorem 3.4 ( $[\mathrm{W}$, $\mathrm{W} 2$, Theorem 1.2]). The bundle $E$ is Gieseker stable if and only if there exist balanced metrics $h^{(k)}$ on $E \otimes L^{k}$ for $k \gg 0$. In addition, if there exists a Hermitian metric $h_{\infty}$ on $E$ such that $h_{k} \rightarrow h_{\infty}$ in $C^{\infty}$, then

$$
\frac{i}{2 \pi} \Lambda F_{\left(E, h_{\infty}\right)}+\frac{1}{2} S\left(\omega_{\infty}\right) I_{E}=\left(\frac{d}{V r}+\frac{\bar{s}}{2}\right) I_{E}
$$

where $h_{k}=h^{(k)} \otimes g_{\infty}^{\otimes(-k)}, S\left(\omega_{\infty}\right)$ is the scalar curvature of $\omega_{\infty}$ and $\bar{s}$ is the average of the scalar curvature. Conversely, if $h_{\infty}$ solves (3.2), then there exists a sequence of balanced metrics $h^{(k)}$ on $E \otimes L^{k}$ for $k \gg 0$ and $h_{k} \rightarrow h_{\infty}$ in $C^{\infty}$. 
In the case that the base manifold $X$ has dimension one and the Kähler metric $\omega_{\infty}$ has constant curvature, we prove that the rate of convergence of $h_{k}$ to $h_{\infty}$ is $O\left(k^{-\infty}\right)$.

Theorem 3.5. Let $X$ be a compact Riemann surface and $\omega_{\infty}$ be a Kähler form of constant curvature on $X$. Let a be a positive integer. Suppose that the Hermitian metric $h_{\infty}$ on E satisfies the Hermitian-Einstein equation

$$
\frac{i}{2 \pi} F_{\left(E, h_{\infty}\right)}=\omega_{\infty} I_{E}
$$

Let $h^{(k)}$ be a sequence of balanced metrics on $E \otimes L^{k}$ for $k \gg 0$ and $h_{k}=h^{(k)} \otimes$ $g_{\infty}^{\otimes(-k)}$. If $h_{k} \rightarrow h_{\infty}$, then

$$
\left\|h_{k}-h_{\infty}\right\|_{C^{a}\left(h_{\infty}\right)}=O\left(k^{-\infty}\right) .
$$

The proof follows from Theorem 3.4. Lemma 3.6 and Lemma 3.7

Lemma 3.6. Let $h$ be a Hermitian metric on $E$. Suppose that $E$ is stable and coefficients $A_{1}, \ldots, A_{q}$ in the asymptotic expansion (3.1) are constant endomorphisms of $E$. If $q$ is large enough, then there exists a sequence of balanced metrics $h^{(k)}$ on $E \otimes L^{k}$ for $k \gg 0$ such that

$$
\left\|h-h^{(k)} \otimes g^{\otimes(-k)}\right\|_{C^{a}(h)}=O\left(k^{3+\frac{13 n}{2}+\frac{a}{2}-q}\right) .
$$

Proof. First we claim that

$$
B_{k}(h)=\frac{\chi(k)}{r V}\left(I_{E}+\sigma_{k}\right),
$$

where $\left\|\sigma_{k}\right\|_{C^{a}}=O\left(k^{n-q-1}\right)$. This holds since $A_{1}, \ldots, A_{q}$ are constant, and therefore we can show that $B_{k}-C I_{E}=O\left(k^{n-q-1}\right)$. More precisely, we observe that there exists a smooth section $A(x)$ of $\operatorname{End}(E)$ such that

$$
B_{k}(h)=k^{n}+A_{1} k^{n-1}+\ldots+A_{q} k^{n-q}+A(x) k^{n-q-1} .
$$

The bundle $E$ is stable and the $A_{j}$ 's are constant sections of $\operatorname{End}(E)$. Therefore there exist numbers $a_{1}, \ldots, a_{q}$ such that $A_{j}=a_{j} I_{E}$. On the other hand,

$$
\int_{X} \operatorname{tr}\left(B_{k}(h)\right) \frac{\omega_{\infty}^{n}}{n !}=\chi(k) V,
$$

where $V=\int_{X} \frac{\omega_{\infty}^{n}}{n !}$. Thus,

$$
B_{k}(h)-\frac{\chi(k)}{r V} I_{E}=\left(A(x)-\frac{1}{r V} \int_{X} A(x) I_{E}\right) k^{n-q-1} .
$$

Define $\sigma_{k}=\left(A(x)-\frac{1}{r V} \int_{X} A(x) I_{E}\right) k^{n-q-1} ;$ we have

$$
B_{k}(h)=\frac{\chi(k)}{r V}\left(I+\sigma_{k}\right),
$$

where $\left\|\sigma_{k}\right\|_{C^{a}}=O\left(k^{n-q-1}\right)$. Now Wang's argument ([W2, page 276]) concludes the proof since $E$ is stable.

Lemma 3.7. In the situation of Theorem 3.5, all coefficient $A_{i}$ 's are constant. 
Proof. The coefficients of the asymptotic expansion of the Bergman kernel are polynomials of the curvature tensor on the base manifold, curvature tensor on the bundle and their covariant derivatives. As the base is a Riemann surface, the whole curvature tensors on the base manifold and on the bundle are constant. Therefore all coefficients are constant.

Remark 3.8. Note that the existence of the $\operatorname{cscK}$ metric on the base and the Hermitian-Eienstein metric on the bundle imply that $A_{1}$ is constant. However, if the dimension of the base is larger than 1, the whole curvature tensor on the base and on the bundle are not necessarily constant. Therefore for $i>1, A_{i}$ is not necessarily constant.

\section{Constructing Almost Balanced metrics}

The goal of this section is to prove Theorem 1.3. In order to prove Theorem 1.3 . we construct a sequence of almost balanced metrics on $\mathcal{O}_{\mathbb{P} E^{*}}(d) \otimes L^{k}$ (Theorem 4.5) . We start with definition of balanced metrics on polarized manifolds.

Let $(Y, \omega)$ be a compact Kähler manifold of dimension $n$ and $\mathcal{O}(1) \rightarrow Y$ be a very ample line bundle on $Y$ equipped with a Hermitian metric $\sigma$ such that $i \bar{\partial} \partial \log \sigma=\omega$. Since $\mathcal{O}(1)$ is very ample, using global sections of $\mathcal{O}(1)$, we can embed $Y$ into $\mathbb{P}\left(H^{0}(Y, \mathcal{O}(1))^{*}\right)$. A choice of ordered basis $\underline{s}=\left(s_{1}, \ldots, s_{N}\right)$ of $H^{0}(Y, \mathcal{O}(1))$ gives an isomorphism between $\mathbb{P}\left(H^{0}(Y, \mathcal{O}(1))^{*}\right)$ and $\mathbb{P}^{N-1}$. Hence for any such $\underline{s}$, we have an embedding $\iota_{\underline{s}}: Y \hookrightarrow \mathbb{P}^{N-1}$ such that $\iota_{\underline{s}}^{*} \mathcal{O}_{\mathbb{P}^{N}}(1)=\mathcal{O}(1)$. Using $\iota_{\underline{s}}$, we can pull back the Fubini-Study metric and the Fubini-Study Kähler form of the projective space to $\mathcal{O}(1)$ and $Y$, respectively.

Definition 4.1. An embedding $\iota_{\underline{s}}$ is called balanced if

$$
\int_{Y}\left\langle s_{i}, s_{j}\right\rangle_{\iota_{\underline{s}}^{*} h_{\mathrm{FS}}} \frac{\iota_{\underline{s}}^{*} \omega_{\mathrm{FS}}^{n}}{n !}=\frac{V}{N} \delta_{i j},
$$

where $V=\int_{Y} \omega^{n} / n$ !. A Hermitian metric (resp. a Kähler form) is called balanced if it is the pullback $\iota_{\underline{s}}^{*} h_{\mathrm{FS}}$ (resp. $\iota_{\underline{s}}^{*} \omega_{\mathrm{FS}}$ ), where $\iota_{\underline{s}}$ is a balanced embedding.

Remark 4.2. The concepts of balanced metric on holomorphic vector bundles (Definition 3.1) and balanced metric on polarized manifolds (Definition 4.1) are different. In order to find a balanced metric on a holomorphic vector bundle $E \rightarrow X$, we need to fix a Kähler form $\omega_{0}$ on $X$. A Hermitian metric $h$ on $E$ is balanced (more precisely, $\omega_{0}$-balanced) if $B\left(h, \omega_{0}\right)=C I_{E}$, where $C$ is a constant. But in order to find a balanced metric on a polarized manifold $(Y, \mathcal{O}(1))$, we do not need to fix a Kähler form. A positive Hermitian metric $g$ on $\mathcal{O}(1)$ is balanced if $B(g, i \bar{\partial} \partial \log g)$ is constant.

Definition 4.3. A sequence of Hermitian metrics $h_{k}$ on $\mathcal{O}(1) \otimes L^{k}$ and ordered bases $\underline{s}^{(k)}=\left(s_{1}^{(k)}, \ldots, s_{N}^{(k)}\right)$ for $H^{0}\left(Y, \mathcal{O}(1) \otimes L^{k}\right)$ are called almost balanced of order $q$ if for any $k$

$$
\sum\left|s_{i}^{(k)}\right|_{h_{k}}^{2}=1
$$

and

$$
\int_{Y}\left\langle s_{i}^{(k)}, s_{j}^{(k)}\right\rangle_{h_{k}} d v o l_{h_{k}}=D^{(k)} \delta_{i j}+M_{i j}^{(k)}
$$


where $D^{(k)}$ is a scalar so that $D^{(k)} \rightarrow C$ as $k \rightarrow \infty$, where $C$ is a constant and $M^{(k)}$ is a trace-free Hermitian matrix such that $\left\|M^{(k)}\right\|_{\text {op }}=O\left(k^{-q-1}\right)$. Here $\left\|M^{(k)}\right\|_{o p}$ is the operator norm of the matrix $M^{(k)}$.

For the rest of this section, let $X$ be a compact Riemann surface and $L$ be an ample line bundle on $X$. Let $g$ be a positive Hermitian metric on $L$ and $\omega_{\infty}=$ $i \bar{\partial} \partial \log g$ be a Kähler form on $X$. Let $E$ be a holomorphic vector bundle on $X$ of rank $r$ and slope $\mu$. The slope of $E$ is defined by $\mu=\frac{\operatorname{deg}(E)}{r}$.

Similar to the case of vector spaces, we have the natural isomorphism $H^{0}\left(\mathbb{P} E^{*}\right.$, $\left.\mathcal{O}_{\mathbb{P} E^{*}}(d) \otimes L^{k}\right)=H^{0}\left(X, \operatorname{Sym}^{d} E \otimes L^{k}\right)$. Also, any Hermitian metric $H$ on $\operatorname{Sym}^{d} E$ induces a Hermitian metric $\widehat{H}$ on $\mathcal{O}_{\mathbb{P} E^{*}}(d) \otimes L^{k}$.

Suppose that $H$ is a Hermitian metric on $\operatorname{Sym}^{d} E$ and $s_{1}, \ldots, s_{N}$ is an orthonormal basis for $H^{0}\left(X, \operatorname{Sym}^{d} E \otimes L^{k}\right)$ with respect to $L^{2}\left(H_{k}, \omega_{\infty}\right)$, where $H_{k}=H \otimes g^{\otimes k}$. Let $\hat{s_{1}}, \ldots, \hat{s_{N}}$ be the corresponding basis for $H^{0}\left(\mathbb{P} E^{*}, \mathcal{O}_{\mathbb{P} E^{*}}(d)\right)$.

We prove that the matrix $\left[\int_{\mathbb{P} E^{*}}\left\langle\widehat{s}_{i}, \widehat{s}_{j}\right\rangle_{\widehat{H}} d v o l_{\widehat{H}}\right]$ is close to a scalar matrix. More precisely, we prove the following.

Proposition 4.4. Let $h_{\infty}$ be a Hermitian-Eienstein metric on E, i.e.

$$
i F_{\left(\bar{\partial}_{E}, h_{\infty}\right)}=\mu \omega_{\infty} I_{E},
$$

where $F_{\left(\bar{\partial}_{E}, h_{\infty}\right)}$ is the curvature of the chern connection of $h_{\infty}$ and $\mu$ is the slope of $E$. Then there exists a constant $C$ that depends only on $r$ and $d$ such that if

$$
\left\|H-\operatorname{Sym}^{d} h_{\infty}\right\|_{C^{2}\left(S y m^{d} h_{\infty}\right)} \leq \min \left(\epsilon, \frac{1}{2}\right),
$$

then

$$
\left|\int_{\mathbb{P} E^{*}}\left\langle\widehat{s_{i}}, \widehat{s_{j}}\right\rangle_{\widehat{H_{k}}} d v o \widehat{C}_{\widehat{H_{k}}}-C_{r, d}(d \mu+k) \delta_{i j}\right| \leq C \epsilon(d \mu+k) .
$$

Here $H_{k}=H \otimes g^{\otimes k}$.

Proof. In this proof $C$ denotes a constant that depends only on $r$ and $d$ that might change from line to line. Define $H_{\infty}=\operatorname{Sym}^{d} h_{\infty}, \omega_{0}=i \bar{\partial} \partial \log \widehat{H_{\infty}}$ and $\omega_{k}=$ $\omega_{0}+k \omega_{\infty}$. Lemma 2.2 implies that $\widehat{H_{\infty}}=\widehat{h_{\infty}} \otimes d$, and therefore $\omega_{0}=d i \bar{\partial} \partial \log \widehat{h_{\infty}}=$ $d \omega_{\widehat{h_{\infty}}}$. A simple calculation shows that $\omega_{\widehat{h_{\infty}}}^{r}=r \mu \omega_{\widehat{h_{\infty}}}^{r-1} \wedge \omega_{\infty}$, since $h_{\infty}$ satisfies the Hermitian-Einstein equation (4.1). Thus,

$$
\omega_{k}^{r}=\omega_{0}^{r}+r k \omega_{0}^{r-1} \wedge \omega_{\infty}=r(d \mu+k) \omega_{0}^{r-1} \wedge \omega_{\infty} .
$$

Therefore,

$$
\begin{aligned}
& \left|\int_{\mathbb{P} E^{*}}\left\langle\widehat{s_{i}}, \widehat{s_{j}}\right\rangle_{\widehat{H_{k}}} \frac{\omega_{k}^{r}}{r !}-C_{r, d}(d \mu+k) \delta_{i j}\right| \\
& \quad=\left|(d \mu+k) \int_{\mathbb{P} E^{*}}\left\langle\widehat{s_{i}}, \widehat{s_{j}}\right\rangle_{\widehat{H_{k}}} \frac{\omega_{0}^{r-1}}{(r-1) !} \wedge \omega_{\infty}-C_{r, d}(d \mu+k) \delta_{i j}\right| \\
& \quad=(d \mu+k)\left|\int_{X}\left(d^{r-1} \int_{\mathbb{P} E_{x}^{*}}\left\langle\widehat{s_{i}}, \widehat{s_{j}}\right\rangle_{\widehat{H_{k}}} \frac{\omega_{\widehat{h_{\infty}}}^{r-1}}{(r-1) !}-C_{r, d} \delta_{i j}\right) \wedge \omega_{\infty}\right| \\
& \quad \leq C(d \mu+k) \epsilon \int_{X}\left|s_{i}\right|_{H_{k}}\left|s_{j}\right|_{H_{k}} \omega_{\infty} .
\end{aligned}
$$


The last inequality follows from Proposition 2.7. Hence, the Cauchy-Schwartz inequality implies that

$$
\begin{aligned}
& \left|\int_{\mathbb{P} E^{*}}\left\langle\widehat{s_{i}}, \widehat{s_{j}}\right\rangle_{\widehat{H_{k}}} \frac{\omega_{k}^{r}}{r !}-C_{r, d}(d \mu+k) \delta_{i j}\right| \\
& \quad \leq C(d \mu+k) \epsilon\left(\int_{X}\left|s_{i}\right|_{H_{k}}^{2} \omega_{\infty}\right)^{\frac{1}{2}}\left(\int_{X}\left|s_{j}\right|_{H_{k}}^{2} \omega_{\infty}\right)^{\frac{1}{2}}=C(d \mu+k) \epsilon,
\end{aligned}
$$

since $s_{1}, \ldots, s_{N}$ is an orthonormal basis for $H^{0}\left(X, \operatorname{Sym}^{d} E \otimes L^{k}\right)$ with respect to $L^{2}\left(H_{k}, \omega_{\infty}\right)$.

On the other hand, Lemma 2.5 implies that $\left\|\omega-\omega_{0}\right\|_{C^{0}\left(\omega_{0}\right)} \leq C \epsilon$. Therefore, $\left\|\left(\omega+k \omega_{\infty}\right)-\omega_{k}\right\|_{C^{0}\left(\omega_{k}\right)}=\left\|\left(\omega+k \omega_{\infty}\right)-\left(\omega_{0}+k \omega_{\infty}\right)\right\|_{C^{0}\left(\omega_{k}\right)} \leq\left\|\omega-\omega_{0}\right\|_{C^{0}\left(\omega_{0}\right)} \leq C \epsilon$, since $\omega_{\infty}$ is a semipositive $(1,1)$-form on $\mathbb{P} E^{*}$. Applying Lemma 2.6 implies that

$$
\left|d v o l_{\widehat{H_{k}}}-\frac{\omega_{k}^{r}}{r !}\right| \leq C \epsilon \frac{\omega_{k}^{r}}{r !} .
$$

Thus,

$$
\begin{aligned}
\left|\int_{\mathbb{P} E^{*}}\left\langle\widehat{s_{i}}, \widehat{s_{j}}\right\rangle_{\widehat{H_{k}}} d v o l_{\widehat{H_{k}}}-\int_{\mathbb{P} E^{*}}\left\langle\widehat{s_{i}}, \widehat{s_{j}}\right\rangle_{\widehat{H_{k}}} \frac{\omega_{k}^{r}}{r !}\right| \leq \int_{\mathbb{P} E^{*}}\left|\left\langle\widehat{s_{i}}, \widehat{s_{j}}\right\rangle_{\widehat{H_{k}}}\right|\left|d v o l_{\widehat{H_{k}}}-\frac{\omega_{k}^{r}}{r !}\right| \\
\leq C \epsilon \int_{\mathbb{P} E^{*}}\left|s_{i}\right|_{H_{k}}\left|s_{j}\right|_{H_{k}} \frac{\omega_{k}^{r}}{r !} \\
\leq C(d \mu+k) \epsilon \int_{\mathbb{P} E^{*}}\left|s_{i}\right|_{H_{k}}\left|s_{j}\right|_{H_{k}} \frac{\omega_{0}^{r-1}}{(r-1) !} \wedge \omega_{\infty} \\
=C(d \mu+k) \epsilon \int_{X}\left|s_{i}\right|_{H_{k}}\left|s_{j}\right|_{H_{k}} \omega_{\infty} \\
\leq C \epsilon\left(\int_{X}\left|s_{i}\right|_{H_{k}}^{2} \omega_{\infty}\right)^{\frac{1}{2}}\left(\int_{X}\left|s_{j}\right|_{H_{k}}^{2} \omega_{\infty}\right)^{\frac{1}{2}} \leq C \epsilon .
\end{aligned}
$$

Here we used (4.3), (4.4) and the fact that $\sup _{\mathbb{P} E_{x}^{*}}\left|\widehat{s}_{i}\right|_{\widehat{H_{k}}}=\left|s_{i}(x)\right|_{H_{k}}$. We have

$$
\begin{aligned}
& \left|\int_{\mathbb{P} E^{*}}\left\langle\widehat{s_{i}}, \widehat{s_{j}}\right\rangle_{\widehat{H_{k}}} d v o l_{\widehat{H_{k}}}-C_{r, d}(d \mu+k) \delta_{i j}\right| \\
& \quad \leq \int_{\mathbb{P} E^{*}}\left|\left\langle\widehat{s_{i}}, \widehat{s_{j}}\right\rangle_{\widehat{H_{k}}}\right|\left|d v o l_{\widehat{H_{k}}}-\frac{\omega_{k}^{r}}{r !}\right|+\left|\int_{\mathbb{P} E^{*}}\left\langle\widehat{s_{i}}, \widehat{s_{j}}\right\rangle_{\widehat{H_{k}}} \frac{\omega_{k}^{r}}{r !}-C_{r, d}(d \mu+k) \delta_{i j}\right| \\
& \quad \leq C(d \mu+k) \epsilon .
\end{aligned}
$$

Theorem 4.5. Let $X$ be a compact Riemann surface and $L \rightarrow X$ be an ample line bundle equipped with a Hermitian metric $g$. Suppose that $i \bar{\partial} \partial \log g=\omega_{\infty}$ is a Kähler form on $X$. Let $E$ be a stable holomorphic vector bundle of rank $r$ on $X$ and $h_{\infty}$ be a Hermitian-Einstein metric on $E$. Let $R$ be the rank of Sym ${ }^{d} E$. Suppose that $\left\{s_{i}^{(k)}\right\}_{i=1}^{N_{k}}$ is a sequence of bases for $H^{0}\left(X, S_{y m}^{d} E \otimes L^{\otimes k}\right)$ and $H^{(k)}$ is a sequence of Hermitian metrics on $S_{y m}{ }^{d} E \otimes L^{\otimes k}$ such that

$$
\begin{aligned}
& \sum_{i=1}^{N_{k}} s_{i}^{(k)} \otimes\left(s_{i}^{(k)}\right)^{{ }^{*} H^{(k)}}=I_{S y m^{d} E \otimes L^{k}}, \\
& \int_{X}\left\langle s_{i}^{(k)}, s_{j}^{(k)}\right\rangle_{H^{(k)}} \omega_{\infty}=\frac{R V \operatorname{ol}\left(X, \omega_{\infty}\right)}{N_{k}} \delta_{i j}, \\
& \left\|H^{(k)} \otimes g^{\otimes(-k)}-S_{y m^{d}} h_{\infty}\right\|_{C^{2}\left(\text { Sym }^{d} h_{\infty}\right)}=O\left(k^{-\infty}\right) .
\end{aligned}
$$


Then the sequence of Hermitian metrics $H^{(k)}$ on $\mathcal{O}_{\mathbb{P} E^{*}}(d) \otimes L^{k}$ and ordered bases $\left.\underline{s}^{(k)}=\widehat{\left(s_{1}^{(k)}\right.}, \ldots, \widehat{s_{N_{k}}^{(k)}}\right)$ of $H^{0}\left(\mathbb{P} E^{*}, \mathcal{O}_{\mathbb{P} E^{*}}(d) \otimes L^{k}\right)$ is almost balanced of order $q$ for any positive integer $q$ in the sense of Definition 4.3 .

Proof. Let $p$ be a positive integer. There exists a constant $C$ independent of $k$ such that

$$
\left\|H^{(k)} \otimes g^{\otimes(-k)}-\operatorname{Sym}^{d} h_{\infty}\right\|_{C^{2}\left(\operatorname{Sym}^{d} h_{\infty}\right)} \leq C k^{-p} .
$$

Fix $k \gg 0$. The basis $\left\{\sqrt{R^{-1} N_{k}} s_{1}^{(k)}, \ldots, \sqrt{R^{-1} N_{k}} s_{N_{k}}^{(k)}\right\}$ is an orthonormal basis for $H^{0}\left(X, \operatorname{Sym}^{d} E \otimes L^{k}\right)$ with respect to $L^{2}\left(H^{(k)}, \omega_{\infty}\right)$. Applying Proposition 4.4 to $H=H^{(k)} \otimes g^{\otimes-k}$ implies that

$$
\left|\int_{\mathbb{P} E^{*}}\left\langle\widehat{s_{i}^{(k)}}, \widehat{s_{j}^{(k)}}\right\rangle_{H^{(k)}} d v o l \widehat{H^{(k)}}-C_{r, d}(d \mu+k) \delta_{i j}\right| \leq C k^{-p}(d \mu+k) .
$$

Define

$$
\begin{aligned}
M^{(k)} & =\int_{\mathbb{P} E^{*}}\left\langle\widehat{s_{i}^{(k)}}, \widehat{s_{j}^{(k)}}\right\rangle_{\widehat{H^{(k)}}} d v o l_{\widehat{H^{(k)}}}-C_{r, d}(d \mu+k) \delta_{i j}, \\
D^{(k)} & =C_{r, d}(d \mu+k) .
\end{aligned}
$$

We have

$$
\int_{\mathbb{P} E^{*}}\left\langle\widehat{s_{i}^{(k)}}, \widehat{s_{j}^{(k)}}\right\rangle_{H^{(k)}} d v o l \widehat{H^{(k)}}=D^{(k)} I+M^{(k)} .
$$

A simple calculation shows that

$$
D^{(k)} \rightarrow C_{r, d} \quad \text { as } k \rightarrow \infty .
$$

On the other hand, (4.5) implies

$$
\left\|M^{(k)}\right\|_{o p} \leq \sum_{i j}\left|\left(M^{(k)}\right)_{i j}\right| \leq C k^{-p}(d \mu+k) N_{k}^{2} \leq C^{\prime} k^{3-p} .
$$

Note that $N_{k}=O(k)$ by the Riemann-Roch theorem. Therefore for any positive integer $q,\left\|M^{(k)}\right\|=O\left(k^{-q-1}\right)$. This means that the sequence of Hermitian metrics $H^{(k)}$ on $\mathcal{O}_{\mathbb{E}^{*}}(d) \otimes L^{k}$ and ordered bases $\left.\underline{s}^{(k)}=\widehat{\left(s_{1}^{(k)}\right.}, \ldots, \widehat{s_{N_{k}}^{(k)}}\right)$ of $H^{0}\left(\mathbb{P} E^{*}, \mathcal{O}_{\mathbb{P} E^{*}}(d) \otimes\right.$ $\left.L^{k}\right)$ is almost balanced of order $q$ for any positive integer $q$.

Proof of Theorem 1.3. Fix a positive integer $a \geq 4$. Let $\omega_{\infty}$ be the Kähler form on $X$ with constant curvature. Since $E$ is a stable bundle, there exists a Hermitian metric $h_{\infty}$ on $E$ that satisfies the Hermitian-Einstein equation (4.1). Therefore Theorem 3.4 and Theorem 3.5 imply that there exists a sequence of balanced metrics $H^{(k)}$ on $\operatorname{Sym}^{d} E \otimes L^{k}$ such that

$$
\left\|H^{(k)} \otimes g^{\otimes(-k)}-\operatorname{Sym}^{d} h_{\infty}\right\|_{C^{a}\left(\operatorname{Sym}^{d} h_{\infty}\right)}=O\left(k^{-\infty}\right) .
$$

By the definition of balanced metrics on vector bundles (Definition 3.1), there exists a sequence of bases $\left\{s_{i}^{(k)}\right\}_{i=1}^{N_{k}}$ for $H^{0}\left(X, \operatorname{Sym}^{d} E \otimes L^{\otimes k}\right)$ such that

$$
\begin{aligned}
\sum_{i=1}^{N_{k}} s_{i}^{(k)} \otimes\left(s_{i}^{(k)}\right)^{*} H^{(k)} & =I_{\mathrm{Sym}^{d} E \otimes L^{k}}, \\
\int_{X}\left\langle s_{i}^{(k)}, s_{j}^{(k)}\right\rangle_{H^{(k)}} \omega_{\infty} & =\frac{R \operatorname{Rol}\left(X, \omega_{\infty}\right)}{N_{k}} \delta_{i j},
\end{aligned}
$$


where $R$ is the rank of $\mathrm{Sym}^{d} E$. Hence

$$
\left.\sum_{i=1}^{N_{k}} \widehat{\mid s_{i}^{(k)}}\right|^{2}{\widehat{H^{(k)}}}^{2}=1 .
$$

Define $\omega_{0}=i \bar{\partial} \partial \log \widehat{H_{\infty}}$ and $\widetilde{\omega_{k}}=i \bar{\partial} \partial \log \widehat{H^{(k)}}$. Thus, (44.6) implies

$$
\begin{gathered}
\left\|\widetilde{\omega_{k}}-\omega_{k}\right\|_{C^{a}\left(\omega_{k}\right)} \leq\left\|\left(\widetilde{\omega_{k}}-k \omega_{\infty}\right)-\omega_{0}\right\|_{C^{a}\left(\omega_{0}\right)}=O\left(k^{-\infty}\right), \\
\left|\log \widehat{H^{(k)}}-\log \left(\widehat{H_{\infty}} \otimes g^{\otimes k}\right)\right|_{C^{a+2}}=\left|\log \left(\widehat{H^{(k)}} \otimes g^{\otimes(-k)}\right)-\log \widehat{H_{\infty}}\right|_{C^{a+2}}=O\left(k^{-\infty}\right) .
\end{gathered}
$$

On the other hand, Theorem 4.5 and (4.7) imply that the sequence of Hermitian metrics $\widehat{H^{(k)}}$ on $\mathcal{O}_{\mathbb{P} E^{*}}(d) \otimes L^{k}$ and ordered bases $\underline{s}^{(k)}=\left(\widehat{s_{1}^{(k)}}, \ldots, \widehat{s_{N_{k}}^{(k)}}\right)$ for $H^{0}\left(\mathbb{P} E^{*}, \mathcal{O}_{\mathbb{P} E^{*}}(d) \otimes L^{k}\right)$ are almost balanced of order $q$ for any positive integer $q$. Since $\mathbb{P} E^{*}$ has no nontrivial holomorphic vector fields, we can perturb these almost balanced metrics to get balanced metrics on $\mathcal{O}_{\mathbb{P} E^{*}}(d) \otimes \pi^{*} L^{k}$ for $k \gg 0$ (see $[$, Theorem 4.6]).

\section{ACKNOWLEDGMENT}

The author is sincerely grateful to Richard Wentworth for many helpful discussions and suggestions and for his continuous help, support and encouragement.

\section{REFERENCES}

[BD] D. Burns and P. De Bartolomeis, Stability of vector bundles and extremal metrics, Invent. Math. 92 (1988), no. 2, 403-407. MR.936089 (89d:53114)

[C] D. Catlin, The Bergman kernel and a theorem of Tian, in Analysis and geometry in several complex variables (Katata, 1997), 1-23, Birkhäuser Boston, Boston, MA, 1999. MR 1699887 (2000e:32001)

[D1] S. K. Donaldson, A new proof of a theorem of Narasimhan and Seshadri, J. Differential Geom. 18 (1983), no. 2, 269-277. MR710055 (85a:32036)

[D2] S. K. Donaldson, Anti self-dual Yang-Mills connections over complex algebraic surfaces and stable vector bundles, Proc. London Math. Soc. (3) 50 (1985), no. 1, 1-26. MR765366 (86h:58038)

[D3] S. K. Donaldson, Scalar curvature and projective embeddings. I, J. Differential Geom. 59 (2001), no. 3, 479-522. MR1916953 (2003j:32030)

[D4] S. K. Donaldson, Scalar curvature and projective embeddings. II, Q. J. Math. 56 (2005), no. 3, 345-356. MR2161248 (2006f:32033)

[DZ] A. Della Vedova and F. Zuddas, Scalar curvature and asymptotic Chow stability of projective bundles and blowups. Trans. Amer. Math. Soc. 364 (2012), no. 12, 6495-6511. MR2958945

[G] D. Gieseker, On the moduli of vector bundles on an algebraic surface, Ann. of Math. (2) 106 (1977), no. 1, 45-60. MR466475(81h:14014)

[H1] Y.-J. Hong, Ruled manifolds with constant Hermitian scalar curvature, Math. Res. Lett. 5 (1998), no. 5, 657-673. MR1666868 (2000j:32039)

[H2] Y.-J. Hong, Constant Hermitian scalar curvature equations on ruled manifolds, J. Differential Geom. 53 (1999), no. 3, 465-516. MR1806068(2001k:32041)

[H3] Y.-J. Hong, Gauge-fixing constant scalar curvature equations on ruled manifolds and the Futaki invariants, J. Differential Geom. 60 (2002), no. 3, 389-453. MR1950172 (2004a:53040)

[L] H. Luo, Geometric criterion for Gieseker-Mumford stability of polarized manifolds, J. Differential Geom. 49 (1998), no. 3, 577-599. MR.1669716 (2001b:32035)

[M] I. Morrison, Projective stability of ruled surfaces, Invent. Math. 56 (1980), no. 3, 269-304. MR561975 (81c:14007)

[PS] D. H. Phong and J. Sturm, Stability, energy functionals, and Kähler-Einstein metrics, Comm. Anal. Geom. 11 (2003), no. 3, 565-597. MR2015757(2004k:32041) 
[PS2] D. H. Phong and Jacob Sturm, Scalar curvature, moment maps, and the Deligne pairing. Amer. J. Math. 126 (2004), no. 3, 693-712. MR2058389 (2005b:53137)

[RT] J. Ross and R. Thomas, An obstruction to the existence of constant scalar curvature Kähler metrics, J. Differential Geom. 72 (2006), no. 3, 429-466. MR2219940 (2007c:32028)

[S] R. Seyyedali, Balanced metrics and Chow stability of projective bundles over Kähler manifolds, Duke Math. J. 153 (2010), no. 3, 573-605. MR2667426 (2012a:32028)

[S2] R. Seyyedali, Balanced metrics and Chow stability of projective bundles over Kähler manifolds. II, preprint.

[UY] K. Uhlenbeck and S.-T. Yau, On the existence of Hermitian-Yang-Mills connections in stable vector bundles, Comm. Pure Appl. Math. 39 (1986), no. S, suppl., S257-S293. MR.861491 (88i:58154)

[W] X. Wang, Balance point and stability of vector bundles over a projective manifold, Math. Res. Lett. 9 (2002), no. 2-3, 393-411. MR1909652 (2004f:32034)

[W2] Xiaowei Wang, Canonical metrics on stable vector bundles. Comm. Anal. Geom. 13 (2005), no. 2, 253-285. MR 2154820 (2006b:32031)

[Z] S. Zelditch, Szegő kernels and a theorem of Tian, Internat. Math. Res. Notices 1998, no. 6, 317-331. MR:1616718 (99g:32055)

[Zh] S. Zhang, Heights and reductions of semi-stable varieties, Compositio Math. 104 (1996), no. 1, 77-105. MR1420712 (97m:14027)

Department of Mathematics, University of California, Irvine, California 92697

E-mail address: rseyyeda@math.uci.edu

Current address: Department of Pure Mathematics, University of Waterloo, 200 University

Avenue West, Waterloo, Ontario, Canada N2L 3G1

E-mail address: rseyyedali@uwaterloo.ca 